\title{
Dolutegravir/rilpivirine for the treatment of HIV-I infection
}

This article was published in the following Dove Press journal: HIVIAIDS - Research and Palliative Care

\section{Ellen Dowers' \\ Francis Zamora' \\ Lydia Aoun Barakat ${ }^{2}$ \\ Onyema Ogbuagu ${ }^{2}$}

'Department of Pharmacy Services, Yale-New Haven Hospital, New Haven, CT, USA; ${ }^{2}$ Yale AIDS Program, Section of Infectious Diseases, Yale University School of Medicine, New Haven, CT, USA
Correspondence: Onyema Ogbuagu Yale AIDS Program, Section of Infectious Diseases, Yale University School of Medicine, New Haven, CT, USA

$\mathrm{Tel}+\mathrm{I} 5 \mathrm{I} 628 \mathrm{I} 4557$

$\mathrm{Fax}+\mathrm{I} 2036883216$

Email onyema.ogbuagu@yale.edu

\begin{abstract}
Much progress has been made in the development of antiretroviral therapies (ARTs) for HIV-1 infection. Beginning a little over a decade ago, single tablet combination regimens (STRs) became available, and subsequently, newer STR formulations with improved safety profiles have emerged. Recently, there is a growing interest in regimen simplification with the primary goal of further reducing long-term toxicities of ART and improving medication adherence. Dolutegravir/rilpivirine (DTG/RPV) was approved by the US Food and Drug Administration (FDA) as the first dual antiretroviral STR for the maintenance therapy of HIV-1 infection. Following an extensive review of all published papers on RPV and DTG, administered alone and in combination, extracted from databases including PubMed, Google scholar, and EMBASE, as well as drug package inserts and conference abstracts and proceedings, this review discusses the chemical properties and composition, pharmacodynamics and pharmacokinetic properties, clinical trial efficacy and safety data, as well as important drug-drug interactions associated with DTG/RPV. An expert opinion section discusses ideal candidates for DTG/ RPV in the context of available but limited data and in comparison to currently available and emerging ART alternatives.
\end{abstract}

Keywords: dolutegravir, rilpivirine, antiretroviral therapy, HIV

\section{Introduction}

More than 37 million people are living with HIV infection worldwide, with about 2 million new infections occurring every year, and, in USA, about 40,000 new infections occur annually, adding to 1.2 million individuals already living with HIV infection. ${ }^{1-3}$ Significant milestones have occurred in the treatment of HIV infection since the first antiretroviral agents became available. Many antiretroviral medications exist today and, when taken consistently, result in sustained and durable viral suppression.

For over 20 years, the paradigm of combination antiretroviral therapy (cART) has been the use of at least three antiretroviral agents with two nucleoside/nucleotide reverse transcriptase inhibitors (NRTIs) as the backbone. ${ }^{4,5}$ In the absence of a cure, the current expectation is lifelong use of cART for HIV disease treatment. However, concern exists regarding long-term toxicities, particularly of NRTIs including their effects on bone mineral density, renal and cardiovascular system toxicities, increased risk for lactic acidosis, and peripheral neuropathy. ${ }^{6-9}$ Recent antiretroviral therapy (ART) constructs have tried to address these concerns, such as the prodrug tenofovir alafenamide that has less renal and bone toxicities than tenofovir disoproxil fumarate (TDF), although it retains some of the other adverse effects (AEs). 
Nonetheless, because of the aforementioned concerns, there is an increasing interest in cART regimen constructs without NRTIs or utilizing simplified two-drug treatment regimens. Multiple studies have evaluated these approaches in both viremic and virologically suppressed HIV-infected patients. ${ }^{10-13}$ Although two-drug regimens have not always performed well in ART-naive individuals, ${ }^{8}$ maintenance or switch therapies in those who have achieved viral suppression on conventional regimens present an effective option with demonstrated efficacy in more recent well-powered studies, although in some cases, associated with the risk of viral resistance. Results of the LATTE and GEMINI studies showed that dual therapy with weekly injectable cabotegravir and rilpivirine (RPV; LATTE), and dolutegravir (DTG) and lamivudine (GEMINI) effectively maintained viral suppression. ${ }^{11-13}$ Although not yet approved for clinical use, these present future viable alternative treatment approaches for patients, particularly those who have achieved full virologic suppression on three-drug regimens. ${ }^{14,15}$

DTG/RPV (Juluca ${ }^{\circledR}$, GlaxoSmithKline, Research Triangle Park, NC, USA) is the first-ever US Food and Drug Administration (FDA) approved dual-drug regimen indicated as maintenance therapy for select HIV-1 infected individuals who are virologically suppressed. Currently, it consists of two medications approved by the FDA for HIV treatment as components of cART - DTG and RPV. ${ }^{16}$ The potency, safety, and high resistance barrier of DTG and the efficacy, safety, and tolerability of RPV along with the favorable pharmacokinetic (PK) profiles of both agents make them good candidates for co-formulation. This nucleoside (tide)-sparing drug combination represents a new treatment approach for the chronic management of the HIV-1-infected patient population.

\section{Overview of the market}

In 1987, zidovudine was the first antiretroviral approved by the US FDA for the treatment of HIV-1 infection..$^{17}$ Thereafter, numerous antiretroviral compounds became available and were subsequently approved as well. Successful combination ART regimens termed highly active antiretroviral therapy (HAART) began in 1996 with the introduction of protease inhibitors (PIs) to the HIV treatment repertoire (first approved in 1995). ${ }^{18}$ The PI class of medications proved to be highly effective against HIV, but typically required multiple pills and doses, constituting a high pill burden for patients. Side effects were common, and regimens were often poorly tolerated. ${ }^{18}$ The introduction of STRs in 2006 (efavirenz [EFV]/TDF and emtricitabine [FTC], Atripla $^{\circledR}$, Gilead Sciences, Inc., Foster City, CA, USA) was a significant breakthrough and advancement in HIV therapeutics allowing for utilization of one pill taken once daily. ${ }^{19}$
Currently, there are over 40 antiretroviral medications grouped into seven different categories according to their mechanism of actions. Antiretroviral regimens for most people living with HIV consist of two NRTIs in combination with a third active drug from one of the following three categories: an integrase strand transfer inhibitor (INSTI), a nonnucleoside reverse transcriptase inhibitor (NNRTI), or a PI with or without a PK enhancer (booster).$^{20}$ The Department of Health and Human Service (DHHS) panel now recommends the use of INSTI-based regimens for initial treatment as evidence shows durable efficacy, limited drug interaction, and toxicity profiles, as well as relative tolerability of these drugs. ${ }^{21}$

In 2017, another advancement in HIV treatment occurred, which was the approval of DTG/RPV by the FDA, and is the first two-drug regimen indicated for chronic maintenance therapy of HIV-1 infection. This new NRTI-sparing regimen represents a well-tolerated treatment option with potential for less long-term toxicity and will be the focus of this review.

\section{Introduction to compound}

DTG/RPV is a two-drug combination of DTG, an INSTI, and RPV, an HIV-1 NNRTI, and is approved as a regimen for the maintenance treatment of HIV-1 infection for select individuals. $^{16}$

\section{Chemistry (DTG)}

DTG is a second-generation integrase inhibitor with chiral, nonracemic structure with a molecular weight of $419 \mathrm{~g} / \mathrm{mol}$. The chemical name of DTG sodium is sodium (4R,12aS)9- $\{[(2,4$-difluorophenyl)methyl $]$ carbamoyl $\}$-4-methyl6,8-dioxo-3,4,6,8,12,12a-hexahydro-2H-pyrido[1',2':4,5] pyrazino[2,1-b][1,3]oxazin-7-olate. The empirical formula is $\mathrm{C}_{20} \mathrm{H}_{18} \mathrm{~F}_{2} \mathrm{~N}_{3} \mathrm{NaO}_{5}$ (Figure 1). This medication is a white light yellow powder that is slightly soluble in water. Each coated tablet of $52.6 \mathrm{mg}$ of DTG sodium is equivalent to 50 mg of DTG.

In addition, the tablet includes D-mannitol, microcrystalline cellulose, povidone K29/32, sodium starch 343 glycolate, and sodium stearyl fumarate as well as iron oxide yellow,

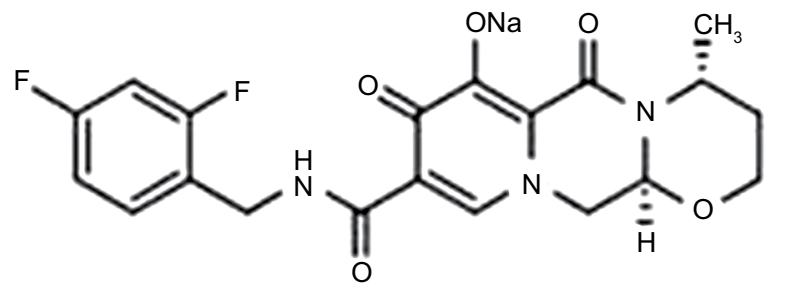

Figure I Chemical structure of dolutegravir. Note: Reproduced from Pubchem. ${ }^{23}$ 
macrogol/polyethylene glycol (PEG), polyvinyl alcohol-part hydrolyzed, talc, and titanium dioxide..$^{22}$

\section{Pharmacodynamics (DTG)}

DTG binds to the two metal cations $\left(\mathrm{Mg}^{2+}\right)$ at the integrase enzyme active site within the preintegration complex. DTG is highly potent when compared to other integrase inhibitors. The in vitro half-maximal inhibitory concentration $\left(\mathrm{IC}_{50}\right)$ is $1.6 \mathrm{nM}$ (compared to 3.3 and $6 \mathrm{nM}$ for raltegravir [RAL] and elvitegravir [EVG], respectively). DTG dissociates from the integrase-DNA complex significantly slower (mean dissociation constant, $2.7 \times 10^{-6} \mathrm{~s}^{-1}$ ) compared to either RAL or EVG $\left(22 \times 10^{-6} \mathrm{~s}^{-1}\right.$ and $71 \times 10^{-6} \mathrm{~s}^{-1}$, respectively)..$^{24,25}$ The structure of DTG allows for increased entry farther into the pocket vacated by the displaced viral DNA (vDNA) making closer contacts with vDNA, compared with those made by RAL and EVG. Some studies suggest that DTG may change its position in response to structural changes in the active site of RAL-resistant integrase protein. ${ }^{26}$

The half maximal effective concentration $\left(\mathrm{EC}^{50}\right)$ of RAL against wild-type (WT) HIV-1 was $4 \mathrm{nM}$, and viruses carrying Y143R, N155H, and G140S/Q148H mutations caused, respectively, a 50-, 30-, and 400-fold decrease in susceptibility to the drug. However, DTG, which inhibited WT HIV-1 with an $\mathrm{EC}_{50}$ of $1.6 \mathrm{nM}$, retained a high potency against the Y143R, N155H, and G140S/Q148H mutants, with less than fourfold decrease in activity. Drugs that engage the side chain at places other than the enzymatic active site should not possess cross-resistance with any other INSTIs and therefore present a viable alternative in those patients who have developed resistance to other medications in this class. ${ }^{27}$

\section{PKs (DTG)}

Following oral ingestion of DTG, peak plasma concentrations are observed in 2-3 hours. The medication achieves steady state in about 5 days. DTG may be taken with or without food; however, food increased the extent but slowed the rate of absorption of DTG. Low-, moderate-, and high-fat meals increased DTG area under the curve (AUC) $(0-\infty)$ by $33 \%$, $41 \%$, and $66 \%$; increased maximum concentration $\left(\mathrm{C}_{\max }\right)$ by $46 \%, 52 \%$, and $67 \%$; and prolonged $\mathrm{T}_{\max }$ to 3,4 , and 5 hours from 2 hours under fasting conditions, respectively. It is highly protein bound (98.9\%), primarily to human plasma proteins. ${ }^{28}$

DTG accumulates in compartments other than plasma (eg, cerebrospinal fluid); however, the clinical significance of this drug is unknown. DTG is primarily metabolized via UGT1A1 with some contribution from CYP3A with a half-life of 14 hours. After a single oral dose of DTG, 53\% of the total oral dose was excreted unchanged in feces. Thirty-one percent of the total oral dose was excreted in urine, represented by an ether glucuronide of DTG (18.9\% of total dose), a metabolite formed by oxidation at the benzylic carbon $(3.0 \%$ of total dose), and its hydrolytic $\mathrm{N}$, de-alkylation product (3.6\% of total dose). Renal elimination of unchanged drug was low $(<1 \%$ of the dose), and no dose adjustment is required in patients with mild, moderate, or severe renal function. ${ }^{28}$

\section{Chemistry (RPV)}

RPV is a diarylpyrimidine derivative and considered a second-generation NNRTI. The molecular formula for RPV is $\mathrm{C}_{22} \mathrm{H}_{18} \mathrm{~N}_{6} \mathrm{HCl}$ with a molecular weight of $402.88 \mathrm{~g} / \mathrm{mol}$. The chemical name for RPV is 4-[[4-[[4-[(E)-2-cyanoethenyl]2,6-dimethylphenyl]amino]-2-pyrimidinyl]amino]benzonitrile monohydrochloride (Figure 2). Each tablet contains the inactive ingredients such as croscarmellose sodium, lactose monohydrate, magnesium stearate, polysorbate 20 , povidone $\mathrm{K} 30$, and silicified microcrystalline cellulose. The tablet coating contains hypromellose 29,106 mPa.s, lactose monohydrate, PEG 3000, titanium dioxide, and triacetin. ${ }^{29}$

\section{Pharmacodynamics (RPV)}

RPV is a diarylpyrimidine NNRTI of HIV-1 and inhibits HIV-1 replication by noncompetitive inhibition of HIV-1 reverse transcriptase (RT). It is considered a second-generation NNRTI and possesses a higher barrier to resistance when compared to previous drugs in this class. ${ }^{29}$

RPV exhibited activity against laboratory strains of WT HIV-1 in an acutely infected T-cell line with a median $\mathrm{EC}_{50}$ value for HIV- $1_{\text {ІІІв }}$ of $0.73 \mathrm{nM}(0.27 \mathrm{ng} / \mathrm{mL})$. RPV also demonstrated antiviral activity against a broad panel of HIV-1 group $\mathrm{M}$ (subtype A, B, C, D, F, G, and $\mathrm{H}$ ) primary isolates with $\mathrm{EC}_{50}$ values ranging from 0.07 to $1.01 \mathrm{nM}(0.03-0.37$ $\mathrm{ng} / \mathrm{mL}$ ) and was less active against group $O$ primary isolates with $\mathrm{EC}_{50}$ values ranging from 2.88 to $8.45 \mathrm{nM}(1.06-3.10 \mathrm{ng} /$ $\mathrm{mL})$. However, limited activity in cell culture against HIV-2 with a median $\mathrm{EC}_{50}$ value of 5,220 nM (range, 2,510-10,830 $\mathrm{nM}$; 920-3970 ng/mL) was observed. ${ }^{29}$

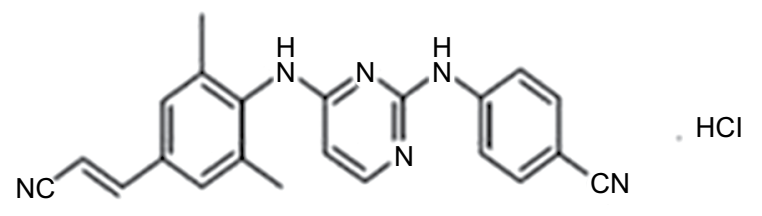

Figure 2 Chemical structure of RPV hydrochloride.

Note: Reproduced from Pubchem. ${ }^{30}$

Abbreviation: RPV, rilpivirine. 


\section{PKs (RPV)}

RPV achieves maximum plasma concentration in 4-5 hours after oral administration. Its absolute bioavailability is unknown; however, exposure to the drug is $40 \%$ lower when taken in fasting conditions compared to coadministration with a fatty or normal caloric meal. ${ }^{31,32}$

RPV is primarily bound to albumin (99.7\%), and its distribution to other compartments of the body (other than plasma) has not been studied. In vitro experiments suggest that it undergoes cytochrome P450 metabolism (CYP3A), and therefore, close attention should be given to other drugs that may affect its elimination.

After a single dose, RPV's half-life is approximately 50 hours. It is excreted primarily in feces accounting for up to $25 \%$ of the administered drug. There is limited or no information regarding the PKs of RPV in patients with moderate or severe renal impairment or in patients with end-stage renal disease (ESRD). The potential impact of altered PKs of RPV in this patient population is expected not to be clinically significant; however, caution should be exercised in patients with poor renal function, and monitoring of possible side effects is warranted..$^{31,32}$

\section{Chemistry of DTG and RPV (in combination)}

In each tablet of DTG/RPV, active ingredients include $50 \mathrm{mg}$ of DTG (equivalent to $52.6 \mathrm{mg}$ DTG sodium) and $25 \mathrm{mg}$ of RPV (equivalent to $27.5 \mathrm{mg}$ RPV hydrochloride). ${ }^{27}$ Inactive ingredients include magnesium stearate, microcrystalline cellulose, silicified microcrystalline cellulose, croscarmellose sodium, sodium starch glycolate, sodium stearyl fumarate D-mannitol, lactose monohydrate, polysorbate 20 , and povidone K29/32 and K30. The film coat of the tablet contains titanium dioxide, macrogol/PEG, part-hydrolyzed polyvinyl alcohol, iron oxide red, iron oxide yellow, and talc, which are also inactive ingredients.

\section{Clinical efficacy}

The clinical efficacy of the individual components - DTG and RPV - were demonstrated in multiple Phase III randomized studies, and both are approved by the FDA individually and in combination with two NRTIs for the treatment of HIV-1 infection. DTG was shown to be superior to comparator regimens in both treatment naive and experienced individuals with approval granted for use in both patient groups in 2013. RPV was studied in treatment naive adults but showed higher virologic failure in subjects with viral loads $>100,000$ copies/ $\mathrm{mL}$ and CD4-T cell counts $<200$ cells $/ \mu \mathrm{L} .{ }^{33-35}$ The approval of RPV was granted in 2011 and limited to treatment naive adults with viral load $>100,000$ and CD4-T cell count $>200$ cells $/ \mu \mathrm{L}$. Efficacy data for the combined use of DTG and RPV come from the pooled analysis of two large parallel randomized controlled trials and smaller observational studies. Details of these trials are summarized in Table 1.

\section{Phase III trials (SWORD-I and 2)}

The safety and efficacy of DTG/RPV were evaluated in two open-label, parallel-group, multicenter, Phase III randomized, noninferiority studies conducted in 12 countries; designated SWORD-1 and SWORD-2. ${ }^{36}$ Eligible patients had to be adults ( $>18$ years of age) on stable ART with HIV-1 RNA levels of at least $<50$ copies $/ \mathrm{mL}$ for at least 6 months and no known major resistance substitutions to any ARV drug class including NNRTI and INSTIs. Subjects were randomly assigned to receive DTG/RPV once daily or to continue their current three drug regimen ART (current antiretroviral therapy [CAR]). The primary endpoint was proportion of participants with HIV-1 RNA lower than 50 copies/mL at week 48 .

Secondary outcomes included other efficacy and safety measures. Efficacy was determined by intention-to-treat analysis at week 24 and week 48 and by subgroups stratified by base ART class.

The change in CD4 cell counts and incidence of observed resistance to DTG or RPV and CAR were determined. Safety endpoints included incidence and severity of adverse events and treatment discontinuation. Laboratory abnormalities and changes in renal, bone, and cardiovascular biomarkers, compared to baseline values, were determined at week 48 .

A total of 1,339 adults in the combined studies were assessed for eligibility, and of these, 1,028 were randomized: 516 to DTG/RPV arm and 512 to CAR arm. The groups were well matched demographically, with equal numbers of patients on PIs, NNRTIs, and INSTIs. The groups were also equally matched in regard to CD4 count (stratified by patients with $<200,200-350,350-500$, and $>500$ cells $/ \mu \mathrm{L}$ ). Prerandomization base ART class was an NNRTI for 54\% in both treatment groups, while $26 \%$ and $27 \%$ were on PIs and $20 \%$ and $19 \%$ on INSTIs for the DTG/RPV and CAR groups, respectively. TDF and FTC were the most frequently used backbone regimens (DTG/RPV group: 73\% TDF and $69 \%$ FTC vs CAR group: 70\% TDF and 67\% FTC).

About equal numbers in each group discontinued treatment or had protocol deviations. Overall, 513 and 511 were included in the intention-to-treat analysis and 457 and 453 subjects were included in the per-protocol analysis for the DTG/RPV and CAR groups, respectively. 


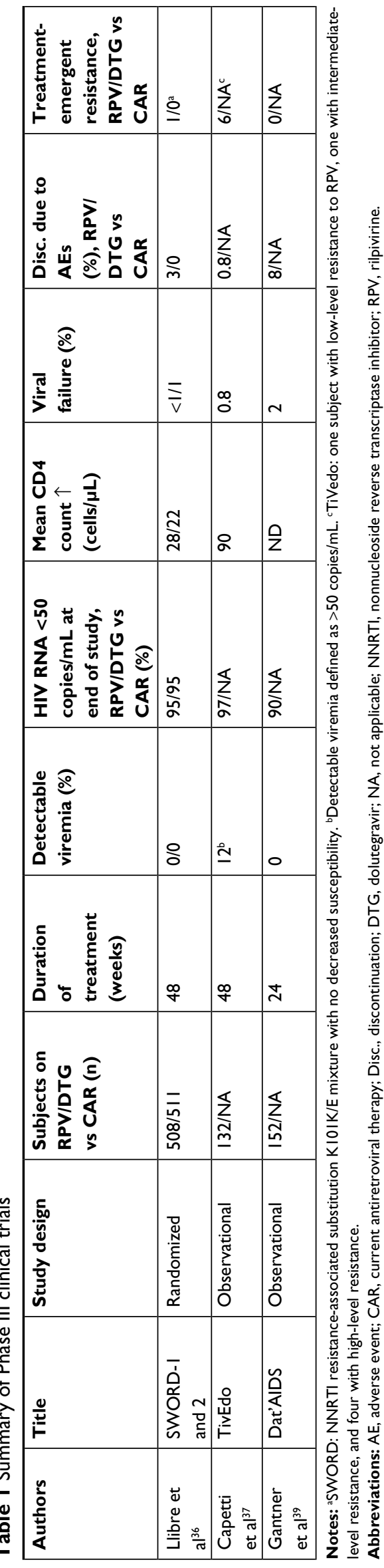

In the pooled analysis of the intention-to-treat population with a set noninferiority margin of $-8 \%, 95 \%$ of subjects in both treatment arms (486 of 513 in the DTG/RPV vs 485 of 511 in the CAR groups) maintained HIV-1 RNA viral loads less than 50 copies $/ \mathrm{mL}$ with an adjusted treatment difference of $-0.2 \%$ ( $95 \% \mathrm{CI},-3.0$ to 2.5 ) confirming noninferiority of DTG/RPV. Treatment efficacy was seen across all subgroups based on the baseline third agent class, CD4 count, and subject demographics. Fewer virologic failures were reported in the DTG/RPV group, which was noninferior to CAR $(0.5 \%$; $95 \% \mathrm{CI},-1.4$ to 0.5$)$ with a predefined noninferiority margin of $4 \%$.

Resistance testing was performed only in subjects who met virologic withdrawal criteria as defined by two consecutive vial loads between 50 and 200 copies per $\mathrm{mL}$. One subject in the DTG/RPV arm showed resistance substitution $\mathrm{K} 101 \mathrm{~K} / \mathrm{E}$ mixture with no decreased susceptibility to RPV. No integrase inhibitor resistance or decreases in DTG susceptibility were seen. Median CD4 cell counts increased from baseline to week 48 by 28 cells $/ \mu \mathrm{L}$ in the DTG/RPV group vs 22 cells $/ \mu \mathrm{L}$ in the CAR group.

Compared with CAR, a nonsignificant decrease in serum lipids was seen in the DTG/RPV group. There was no change in estimated glomerular filtration rate (eGFR) in both groups; bone biomarkers improved significantly in the DTG/RPV group and did not differ from baseline in the CAR group.

\section{Observational study (TiVEdo)}

In the observational cohort study TiVEdo, ART regimens were switched to DTG plus RPV (as separate components) for 132 subjects and followed for up to 48 weeks. ${ }^{37}$ The cohort was composed of $32 \%$ females and $10 \%$ non-Caucasians and had a mean age of 52 years. Reasons for switching included simplification (53\%), toxicity $(34.8 \%)$, drug interactions $(5.3 \%)$, persistent low-level viremia $(3.03 \%)$, nonadherence $(2.27 \%)$, and virologic failure (1.51\%). At baseline, 16 patients had quantifiable detectable virus, 27 had $<50$ HIV-1 RNA copies/mL (below threshold), and 89 had no virus detected (NVD).

Of the 16 subjects with detectable virus, three continued to have detectable viremia ( $>50$ copies $/ \mathrm{mL}$ ) at week 4 . However, by week 24 , only one patient remained with detectable viremia. The below-threshold group decreased from 27 to $19(63 \%)$ and the NVD increased from 89 to $112(81 \%)$ by week 24 . Of the 50 subjects who had a follow-up at 48 weeks, one was off treatment ( $>75 \%$ of time) at study visits and had virologic failure. Four patients remained below threshold and 
45 had NVD (90.9\%). Mean CD4+ T-cell count increased over time for the entire cohort ( $721-811$ cells $/ \mu \mathrm{L})$.

Among all subjects, six patients had some levels of resistance to RPV at baseline, but this did not result in treatment failure (defined as $>50 \mathrm{HIV}-1 \mathrm{RNA}$ copies/mL). No INSTI mutations were detected at baseline. The regimen was well tolerated with only one patient discontinuing the treatment due to headache and insomnia. In addition, one patient terminated the treatment due to drug interaction between RPV and proton pump inhibitors. Only one patient developed some mutations to NNRTIs due to interruptions in therapy leading to virologic failure. The study found that subjects on DTG/ RPV experienced a mean serum creatinine increase by +0.1 $\mathrm{mg} / \mathrm{dL}$. This increase was possibly due to the known effect of DTG, which causes decreased tubular secretion of creatinine via inhibition of renal organic cation transporter 2 (OCT 2). ${ }^{38}$

\section{Observational study (Dat'AIDS)}

The Dat'AIDS cohort was a retrospective analysis of 152 patients with HIV-1 RNA <50 copies/mL whose ART regimen was switched to DTG plus RPV (as separate components). ${ }^{39}$ Subjects were mostly male (66\%) with a median age of 55 years and with viral suppression maintained for a median of 10 years. The primary outcome was a mixed analysis of antiviral efficacy based on the loss of virologic and/or therapeutic response at week 24. Loss of virologic control was defined as two measurements within 4 weeks showing HIV-1 RNA > 50 copies/mL or one plasma sample with HIV-1 RNA $>1,000$ copies $/ \mathrm{mL}$. Therapeutic failure was defined as discontinuation of study medications for any reason. Before dual therapy, $41 \%$ were INSTI naive and $52 \%$ overall had experienced virologic failure on a previous ART regimen.

At week 24, 115 of 116 subjects (99.1\%) did not meet the definition of virologic failure and 105 of 116 (95\%) were without therapeutic failure. At the end of the study, confirmed virologic failure occurred in only three patients ( $2 \%)$. Two of these patients had low-level transient viremia, remained on DTG/RPV, and were found to have WT virus (RNA Sanger sequencing). No resistance testing was done on the third patient who was lost to follow-up.

Overall, 19 patients (13\%) stopped dual therapy with 12 subjects discontinuing the treatment due to adverse effects (AEs), mostly neurologic disorders. Others discontinued the treatment for multiple reasons including simplification with change to a single-tablet regimen $(n=3)$, patients' decision $(n=1)$, virologic failure $(n=1)$, pregnancy $(n=1)$, and drug interaction $(n=1)$. No grade 3 and 4 AEs were observed. There were no significant changes in CD4 count; a median increase in serum creatinine of $+11.1 \pm 5.4 \mu \mathrm{mol} / \mathrm{L}$ occurred by week 24 of the study compared to baseline values ( $P$-value $<0.001)$.

\section{Safety and toxicity of DTG/RPV (Phase III trials)}

AEs were reported in 395 (77\%) subjects in the DTG/RPV and 364 (71\%) subjects in the CAR groups (Table 2). ${ }^{36}$ The most common events were nasopharyngitis, headache, upper respiratory tract infection, diarrhea, and back pain. More participants $-17(3 \%)$ in the DTG/RPV arm withdrew from the study, compared to three $(1 \%)$ in the CAR group due to drug-related events. This higher discontinuation rate in the DTG/RPV arm related to drug intolerability (eg, headache and diarrhea) was expected given that patients in the CAR group were enrolled and maintained on a stable regimen. Neuropsychiatric AEs including insomnia, depression, anxiety, and abnormal dreams occurred more commonly in the DTG/RPV group. These AEs often occurred in subjects with a history of anxiety, depression, or insomnia. The majority of overall events were considered grade 1 in 247 (48\% in both groups) or grade 2 in 116 (23\% in DTG/RPV and 20\% in CAR). Three patients did not have a virologic response to DTG/RPV, compared to six in the CAR group.

\section{Safety and toxicity}

\section{DTG}

The absorption of DTG is significantly impaired by coadministration with divalent or trivalent cations such as iron,

Table 2 Selected adverse effects adapted from Phase III trials (week 48)

\begin{tabular}{|c|c|c|}
\hline Adverse effects & $\begin{array}{l}\text { DTG/RPV } \\
(n=5 \mid 3), n(\%)\end{array}$ & $\begin{array}{l}\text { CAR } \\
(n=5 I I), \\
n(\%)\end{array}$ \\
\hline $\begin{array}{l}\text { Any adverse } \\
\text { effects }\end{array}$ & $395(77)$ & $364(7 I)$ \\
\hline Psychiatric & $61(12)$ & $32(6)$ \\
\hline Nasopharyngitis & $49(10)$ & $50(10)$ \\
\hline Headache & $4 I(8)$ & $23(5)$ \\
\hline Diarrhea & $32(6)$ & $27(5)$ \\
\hline $\begin{array}{l}\text { Drug-related } \\
\text { adverse effects }\end{array}$ & $97(19)$ & $9(2)$ \\
\hline Headache & II (2) & 0 \\
\hline Diarrhea & $8(2)$ & $\mathrm{I}(<\mathrm{I})$ \\
\hline $\begin{array}{l}\text { Serious events } \\
\text { Medication related } \\
\text { Fatal events }\end{array}$ & $\begin{array}{l}27(5) \\
4(1) \\
I(<I)\end{array}$ & $\begin{array}{l}2 I(4) \\
I(<I) \\
I(<I)\end{array}$ \\
\hline $\begin{array}{l}\text { Events leading } \\
\text { to study } \\
\text { withdrawal }\end{array}$ & $17(3)$ & $3(1)$ \\
\hline
\end{tabular}

Note: Date from Llibre et al. ${ }^{36}$

Abbreviations: CAR, current antiretroviral therapy; DTG, dolutegravir; RPV, rilpivirine. 
calcium, aluminum, and magnesium. ${ }^{36}$ For example, AUC decreases by $39 \%$ and by $54 \%$ if DTG is given concomitantly with calcium carbonate and ferrous fumarate, respectively. Coadministration of magnesium/aluminum containing antacids decreases AUC and $\mathrm{C}_{\max }$ by $74 \%$ and $72 \%$, respectively. Such effects are minimized if DTG is taken 2 hours before or 6 hours after these agents. ${ }^{16}$

Drugs that induce CYP 3A4 and transporters (UGT1A1, P-glycoprotein) will reduce DTG serum levels impacting drug efficacy. Such decreases are seen with concomitant administration of rifampin, carbamazepine, or EFV .

The PKs of metformin and dofetilide are altered when given with DTG with resultant increased levels of both drugs, therefore, the recommendation for dose limit restrictions with metformin and the contraindication for concurrent use with dofetilide. ${ }^{16}$

\section{RPV}

Given the pH-dependent absorption of RPV, concomitant administration with agents affecting gastric acidity can alter its levels. RPV administration is contraindicated with proton pump inhibitors and should be given spaced in dosing from histamine 2-receptor antagonists and antacids. ${ }^{16}$

Like DTG, CYP3A4 enzyme inducers including carbamazepine, dexamethasone, hypericum (St. John's wort), phenobarbital, phenytoin, rifampin, and rifabutin decrease RPV plasma concentration and should generally be avoided. However, when used concurrently with rifabutin, an additional 25 mg of RPV may be administered with fixed dose combination of DTG/RPV for the duration of rifabutin treatment (Table 3).

\section{Regulatory affairs}

DTG/RPV received the US FDA approval on November 21, 2017, for maintenance therapy of HIV-1 infection in adults who are virologically suppressed on a stable antiretroviral regimen for at least 6 months and with no history of treatment failure and no known resistance substitutions to its individual components. On March 22, 2018, DTG/RPV received similar authorization for use by the European Medical Association. ${ }^{40}$

\section{Dosing routes}

DTG/RPV is given as one oral tablet daily with a meal.

\section{Conclusion and expert opinion}

DTG/RPV represents an effective option for the long-term treatment of HIV-1 infection. Both agents, as have been described in the earlier sections of this article, have favorable profiles including that they are well tolerated, have limited drug interaction potential, and have been shown in clinical trials to achieve and maintain virologic suppression with low rates of treatment failure and treatment -emergent resistance. ${ }^{41-44}$ Furthermore, the exclusion of the NRTI class of medications is an attractive strategy as it limits known long-term toxicities associated with these agents.

However, as a first-in-class, two-drug ART strategy, providers may be wary of a less than three drug-containing ART regimen for managing their patients. However, the clinical trials data are compelling and should be reassuring for use in selected patients who meet the study eligibility criteria including those with long-term virologic suppression and absence of preexisting resistance mutations to the component

Table 3 Drugs contraindicated for coadministration with DTG/RPV

\begin{tabular}{|l|l|l|}
\hline Drug class & Contraindicated drugs in class & Clinical comment \\
\hline Antiarrhythmic & Dofetilide & $\begin{array}{l}\text { Potential for increased dofetilide plasma } \\
\text { concentrations may lead to serious/life- } \\
\text { threatening cardiac events }\end{array}$ \\
\hline Anticonvulsants & $\begin{array}{l}\text { Carbamazepine } \\
\text { Oxcarbazepine } \\
\text { Phenobarbital } \\
\text { Phenytoin }\end{array}$ & $\begin{array}{l}\text { Induction of CYP3A enzyme by } \\
\text { anticonvulsants and the use of systemic } \\
\text { corticosteroids may result in significant } \\
\text { decreases in RPV plasma concentration, } \\
\text { resulting in virologic failure }\end{array}$ \\
\hline Glucocorticoid (systemic) & $\begin{array}{l}\text { Dexamethasone } \\
\text { (more than a single dose) }\end{array}$ & $\begin{array}{l}\text { St. John's wort } \\
\text { (Hypericum perforatum) }\end{array}$ \\
\hline Herbal products & $\begin{array}{l}\text { Esomeprazole } \\
\text { Lansoprazole } \\
\text { Omeprazole } \\
\text { Pantoprazole }\end{array}$ & $\begin{array}{l}\text { RPV requires an acidic medium (low pH) } \\
\text { for absorption; therefore, agents that } \\
\text { affect gastric pH will impair adequate } \\
\text { absorption and possibly cause virologic } \\
\text { failure }\end{array}$ \\
\hline Proton pump inhibitors & \\
\hline
\end{tabular}

Note: Data from GSK. ${ }^{27}$

Abbreviations: DTG, dolutegravir; RPV, rilpivirine. 
Table 4 Changes in BMD, lipid profile, and GFR with DTG/RPV (at 48 weeks)

\begin{tabular}{|l|l|l|l|}
\hline Study & Biomarkers & eGFR \\
\hline & $\Delta$ BMD & $\begin{array}{l}\Delta \text { Lipids } \\
\text { (HDL/LDL/TG) }\end{array}$ \\
\hline SWORD-I and 2 & & Nonsignificant $\downarrow$ & $\leftrightarrow$ \\
\hline TiVedo $^{37}$ & T-score $\uparrow 1.34$ hip, I.46 spine & ND & Significant $\downarrow$ \\
\hline Dat'AIDS $^{39}$ & ND & ND & ND \\
\hline
\end{tabular}

Note: $\uparrow$, significant increase; $\downarrow$, decrease; $\leftrightarrow$, no significant change.

Abbreviations: BMD, bone mineral density; DTG, dolutegravir; eGFR, estimated glomerular filtration rate; GFR, glomerular filtration rate; HDL, high-density lipoprotein; LDL, low-density lipoprotein; ND, not discussed; RPV, rilpivirine; TG, triglyceride.

drugs. In clinical practice, other ideal candidates for switch to DTG and RPV are those who have developed toxicities related to NRTIs (including those for whom providers desire PIs, or first-generation NNRTIs).

Multiple studies are also supporting that that a less than 3 drug drug strategy may be possible for a maintenance phase of therapy. ${ }^{10-15}$ As these studies have shown, indeed, the integrase inhibitors and some PIs are so potent and have a high barrier to resistance, such that they can result in or maintain full virologic suppression with the addition of a single (other class) antiretroviral agent. Therefore, a three-drug (or two-drug) induction phase and subsequent maintenance therapy with two-drug therapy (or single drug such as with monoclonal antibodies) $)^{45}$ in stably virologically suppressed individuals could be the treatment paradigm of the future.

Some clinical concerns around the regimen are justified including recent reports of neuropsychiatric AEs in patients on DTG and also PK interactions with commonly used agents such as divalent cations contained in multivitamins, antacids, and supplements for treating mineral bone disease ${ }^{46}$ (although dosing can be changed to avoid this), antitubercular agents (an important consideration for tuberculosis [TB] endemic areas), and RPV's vulnerability to diminished absorption with antacids, which are very commonly used. In addition, with the absence of antivirals (in DTG/RPV combination) with activity against hepatitis $\mathrm{B}$ virus (HBV), this regimen may not be an option for those co-infected with HBV.

Although limited data are available, and more long-term studies with more patients are required; the neutral effect on lipids by DTG/RPV following a switch from other cART regimens and the lack of renal and bone toxicity (which has been associated with certain NRTIs) are desirable properties (Table 4). Furthermore, the regimen may be utilized without dose adjustment in patients with renal disease. It is important to mention that although creatinine elevations are noted with DTG, which occurs through its inhibition of renal transporterOCT 2 - this does not reflect a true decline in renal function. ${ }^{38}$

Overall, DTG/RPV has ushered in a new era of maintenance therapy for HIV disease and is an effective, well toler- ated and with potential for comparatively fewer long-term toxicities compared to NRTI containing regimens.

\section{Disclosure}

Lydia Aoun Barakat has served on advisory board for Gilead Sciences, Inc. Onyema Ogbuagu has served on advisory board and speakers' bureau for Gilead Sciences, Inc. Ellen Dowers and Francis Zamora report no conflicts of interest in this work.

\section{References}

1. Centers for Disease Control and Prevention [homepage on the Internet]. HIV Surveillance Report: Diagnoses of HIV Infection in the United States and Dependent Areas; 2016. Available from: https:// www.cdc.gov/hiv/statistics/overview/index.html. Accessed January 18, 2018.

2. UNAIDS. Global report 2013: UNAIDS report on the global AIDS epidemic 2013. Available from: https://www.unaids.org/en/media/ unaids/contentassets/documents/epidemiology/2013/gr2013/UNAIDS_ Global_Report_2013_en.pdf. Accessed January 18, 2018.

3. UNAIDS. AIDS by the numbers. Available from: http://www.unaids. org/en/media/unaids/contentassets/documents/unaidspublication/2013/ JC2571_AIDS_by_the_numbers_en.p. Accessed February 2, 2018.

4. Ambrosioni J, Calmy A, Hirschel B. HIV treatment for prevention. $J$ Int AIDS Soc. 2011;14:28.

5. UNAIDS. Global HIV/AIDS response: epidemic update and health sector progress towards universal access. Progress report 2011. Available from: http://www.unaids.org/en/media/unaids/contentassets/documents/ unaidspublication/2011/20111130_ua_report_en.pdf. Accessed February $10,2018$.

6. Lactic Acidosis International Study Group. Risk factors for lactic acidosis and severe hyperlactataemia in HIV-1-infected adults exposed to antiretroviral therapy. AIDS. 2007;21(18):2455-2464.

7. Arenas-Pinto A, Bhaskaran K, Dunn D, Weller IV. The risk of developing peripheral neuropathy induced by nucleoside reverse transcriptase inhibitors decreases over time: evidence from the Delta trial. Antivir Ther. 2008;13:289-295.

8. Kozal M, Lupo S, DeJesus E, et al. A Nucleoside- and Ritonavir-Sparing Regimen Containing Atazanavir Plus Raltegravir in Antiretroviral Treatment-Naïve HIV-Infected Patients: SPARTAN Study Results. HIV Clin Trials. 2012;13(3):119-130.

9. Sterne JA, Hernán MA, Ledergerber B. Long-term effectiveness of potent antiretroviral therapy in preventing AIDS and death: a prospective cohort study. Lancet. 2005;366(9483):378-384.

10. Taiwo B, Zheng L, Gallien S, et al. Efficacy of a nucleoside-sparing regimen of darunavir/ritonavir plus raltegravir in treatment-naive HIV1-infected patients (ACTG A5262). AIDS. 2011;25(17):2113-2122.

11. Taiwo B, Marconi V. Dolutegravir plus lamivudine maintain human immunodeficiency virus-1 suppression through week 48 in a pilot randomized trial. CID. 2018;11:1794-1797. 
12. Margolis DA, Gonzalez-Garcia J, Stellbrink HJ, et al. Long-acting intramuscular cabotegravir and rilpivirine in adults with HIV-1 infection (LATTE-2): 96-week results of a randomised, open-label, phase 2b, non-inferiority trial. Lancet. 2017;390(10101):1499-1510.

13. Borghetti A, Baldin G, Lombardi F, et al. Efficacy and tolerability of lamivudine plus dolutegravir as a switch strategy in a multicentre cohort of patients with suppressed HIV-1 replication. HIV Med. 2018;19(7):452-454.

14. Pasquau J, Hidalgo-Tenorio C. Nuke-Sparing Regimens for the LongTerm Care of HIV Infection. AIDS Rev. 2015;17(4):220-230.

15. Achhra AC, Boyd MA. Antiretroviral regimens sparing agents from the nucleoside(tide) reverse transcriptase inhibitor class: a review of the recent literature. AIDS Res Ther. 2013;10(1):33.

16. FDA [homepage on the Internet]. FDA approved first two-drug regimen for certain patients with HIV. Available from: https:/www.fda.gov/ newsevents/newsroom/pressannouncements/ucm586305.htm. Accessed February 1, 2018.

17. Mitusa H, Weinhold K. 3'-Azido-3'deoxythimidine (BW A509U): An antiviral agent that inhibits the infectivity and cytopathic effect of human T-lymphothrotic virus type III/lymphadenopathy-associated virus in vitro. Proc Natl Acad Sci. 1985;92:7096-7100.

18. Hammer SM, Squires KE, Hughes MD, et al. A controlled trial of two nucleoside analogues plus indinavir in persons with human immunodeficiency virus infection and CD4 cell counts of 200 per cubic millimeter or less. AIDS Clinical Trials Group 320 Study Team. $N$ Engl J Med. 1997;337(11):725-733.

19. Staszewski S, Morales-Ramirez J, Tashima KT, et al. Efavirenz plus zidovudine and lamivudine, efavirenz plus indinavir, and indinavir plus zidovudine and lamivudine in the treatment of HIV-1 infection in adults. Study 006 Team. N Engl J Med. 1999;341(25):1865-1873.

20. Knechten H, Lutz T, Pulik P, Martin T, Tappe A, Jaeger H. Safety and Efficacy in HIV-1-Infected Patients Treated with Ritonavir-Boosted Saquinavir Mesylate. Arch Drug Inf. 2010;3(1):26-36.

21. Department of Health and Human Services Panel on Antiretroviral Guidelines for Adults and Adolescents. Guidelines for the Use of Antiretroviral Agents in Adults and Adolescents Living with HIV. Available from: http://www.aidsinfo.nih.gov/ContentFiles/AdultandAdolescentGL.pdf. Accessed January 8, 2018.

22. Palella FJ, Delaney KM, Moorman AC, et al. Declining morbidity and mortality among patients with advanced human immunodeficiency virus infection. HIV Outpatient Study Investigators. $N$ Engl J Med. 1998;338(13):853-860.

23. Pubchem. Structure of dolutegravir sodium. Available from: https://pubchem.ncbi.nlm.nih.gov/compound/46216142\#section=Top. Accessed February 8, 2018.

24. Podany AT, Scarsi KK, Fletcher CV. Comparative Clinical Pharmacokinetics and Pharmacodynamics of HIV-1 Integrase Strand Transfer Inhibitors. Clin Pharmacokinet. 2017;56(1):25-40.

25. Kandel C, Walmsley S. DTG - a review of the pharmacology, efficacy, and safety in the treatment of HIV. Drug Des Devel Ther 2015;9:3547-3555.

26. Cottrell ML, Hadzic T, Kashuba AD. Clinical pharmacokinetic, pharmacodynamic and drug-interaction profile of the integrase inhibitor dolutegravir. Clin Pharmacokinet. 2013;52(11):981-994.

27. GSK. Dolutegravir and rilpivirine for Oral use: Highlights of Prescrib-

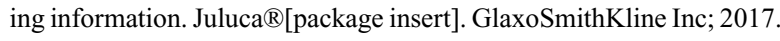
Available from: https://www.gsksource.com/pharma/content/dam/ GlaxoSmithKline/US/en/Prescribing_Information/Juluca/pdf/JULUCAPI-PIL.PDF. Accessed February 10, 2018.

28. GSK. Dolutegravir tablets for oral use: Highlights of Prescribing information.Tivicay®(DTG) [package insert]. Available from: https:// www.gsksource.com/pharma/content/dam/GlaxoSmithKline/US/en/ Prescribing_Information/Tivicay/pdf/TIVICAY-PI-PIL.PDF. Accessed February 10, 2018.

29. Janssen Therapeutics. Rilpivirine tablets for oral use: Highlights of Prescribing Information. Edurant ${ }^{(R P V)}$ [package insert]. Available from: http://www.edurant.com/shared/prescribing-information-edurant. pdf. Accessed February 10, 2018.
30. Pubchem. Structure of rilpivirine hydrochloride. Available from: https://pubchem.ncbi.nlm.nih.gov/compound/11711114\#section=Top. Accessed February 8, 2018.

31. van Heeswijk R, Hoetelmans R. The effects of CYP3A4 modulation on the pharmacokinetics of TMC278, an investigational non-nucleoside reverse transcriptase inhibitor (NNRTI). Poster presented at: The Seventh International Workshop of Clinical Pharmacology; April 20-22, 2006; Lisbon, Portugal.

32. Ford N, Lee J, Andrieux-Meyer I, Calmy A, Safety, efficacy, and pharmacokinetics of rilpivirine: systematic review with an emphasis on resource-limited settings. HIV AIDS. 2011;3:35-44.

33. Ogbuagu O. Rilpivirine, emtricitabine and tenofovir alafenamide: single-tablet combination for the treatment of HIV-1 infection in selected patients. Expert Rev Anti Infect Ther. 2016;14(12):1113-1126.

34. Cohen CJ, Molina JM. Efficacy and safety of rilpivirine (TMC278) versus efavirenz at 48 weeks in treatment-naive HIV-1-infected patients: pooled results from the phase 3 double-blind randomized ECHO and THRIVE Trials. J Aquir Immune Defic Syndr. 2012;1;60(1): 33-42.

35. Crauwels H, Vingerhoets J, Ryan R, Witek J, Anderson D. Pharmacokinetic parameters of once-daily rilpivirine following administration of efavirenz in healthy subjects. Antivir Ther. 2012;17(3):439-446.

36. Llibre JM, Hung CC, Brinson C, et al. Efficacy, safety, and tolerability of dolutegravir-rilpivirine for the maintenance of virological suppression in adults with HIV-1: phase 3, randomised, non-inferiority SWORD-1 and SWORD-2 studies. Lancet. 2018;391(10123):839-849.

37. Capetti AF, Sterrantino G, Cossu MV, et al. Switch to Dolutegravir plus Rilpivirine Dual Therapy in cART-Experienced Subjects: An Observational Cohort. PLoS One. 2016;11(10):e0164753.

38. Lindeman TA, Duggan JM, Sahloff EG. Evaluation of Serum Creatinine Changes With Integrase Inhibitor Use in Human Immunodeficiency Virus-1 Infected Adults. Open Forum Infect Dis. 2016;3(2):ofw053.

39. Gantner P, Cuzin L, Allavena C, et al. Efficacy and safety of dolutegravir and rilpivirine dual therapy as a simplification strategy: a cohort study. HIV Med. 2017;18(9):704-708.

40. Juluca [homepage on the Internet]. European Medicines Agency Human medicines - Juluca. Available from: http:/www.ema.europa.eu/ ema/index.jsp?curl=pages/medicines/human/medicines/004427/smops/ Positive/human_smop_001278.jsp\&mid=WC0b01ac058001d127. Accessed April 23, 2018.

41. Walmsley S, Baumgarten A. Dolutegravir plus abacavir/lamivudine for the treatment of HIV-1 infection in antiretroviral therapy-naive patients: week 96 and week 144 results from the SINGLE randomized clinical trial. J Acquir Immune Defic Syndr. 2015;70:515-519.

42. Raffi F, Jaeger H, Quiros-Roldan E, et al. Once-daily dolutegravir versus twice-daily raltegravir in antiretroviral-naive adults with HIV-1 infection (SPRING-2 study): 96 week results from a randomised, double-blind, non-inferiority trial. Lancet Infect Dis. 2013;13(11):927-935.

43. Molina JM, Clotet B, van Lunzen J, et al. Once-daily dolutegravir versus darunavir plus ritonavir for treatment-naive adults with HIV-1 infection (FLAMINGO): 96 week results from a randomised, open-label, phase 3b study. Lancet HIV. 2015;2(4):e127-e136.

44. Cohen CJ, Andrade-Villanueva J, Clotet B, et al. Rilpivirine versus efavirenz with two background nucleoside or nucleotide reverse transcriptase inhibitors in treatment-naive adults infected with HIV-1 (THRIVE): a phase 3, randomised, non-inferiority trial. Lancet. 2011;378(9787):229-237.

45. Dhody K, Pourhassan N, Kazempour K, et al. PRO 140, a monoclonal antibody targeting CCR5, as a long-acting, single-agent maintenance therapy for HIV-1 infection. HIV Clin Trials. 2018;19(3):85-93.

46. Patel P, Song I, Borland J, et al. Pharmacokinetics of the HIV integrase inhibitor S/GSK1349572 co-administered with acid-reducing agents and multivitamins in healthy volunteers. J Antimicrob Chemother. 2011;66(7):1567-1572. 


\section{Publish your work in this journal}

HIV/AIDS - Research and Palliative Care is an international, peerreviewed open access journal focusing on advances in research in HIV, its clinical progression and management options including antiviral treatment, palliative care and public healthcare policies to control viral spread. The journal is included in PubMed. The manuscript man-

Submit your manuscript here: https://www.dovepress.com/hivaids---research-and-palliative-care-journal 